\title{
Biological and psychological components of depression in patients receiving IFN-alpha therapy for hepatitis C
}

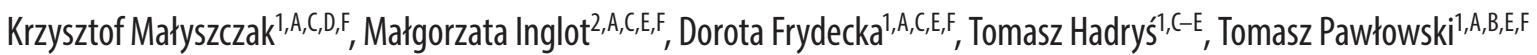 \\ ${ }^{1}$ Department of Psychiatry, Wroclaw Medical University, Poland \\ ${ }^{2}$ Department of Infectious Diseases, Liver Diseases and Acquired Immune Deficiencies, Wroclaw Medical University, Poland \\ A - research concept and design; $\mathrm{B}$ - collection and/or assembly of data; $\mathrm{C}$ - data analysis and interpretation; \\ $D$ - writing the article; $E$ - critical revision of the article; $F$ - final approval of the article
}

\author{
Address for correspondence \\ Krzysztof Małyszczak \\ E-mail: krzysztof.malyszzzak@umed.wroc.pl \\ Funding sources \\ Polish National Science Centre \\ (grant No. N402 586140) \\ Conflict of interest \\ None declared

\section{Acknowledgements} \\ The authors would like to thank prof. Brygida \\ Knysz and prof. Andrzej Gładysz for their support \\ in the organization and design of the study.
}

Received on 0ctober 23, 2018

Reviewed on January 24, 2019

Accepted on February 21, 2019

Published online on February 27, 2019

Cite as

Małyszzzak K, Inglot M, Frydecka D, Hadryś T, Pawłowski T. Biological and psychological components of depression in patients receiving IFN-alpha therapy for hepatitis C. Adv Clin Exp Med. 2019;28(9):1217-1222.

doi:10.17219/acem/104617

DOI

10.17219/acem/104617

\section{Copyright}

Copyright by Author(s)

This is an article distributed under the terms of the

Creative Commons Attribution Non-Commercial License

(http://creativecommons.org/licenses/by-nc-nd/3.0/)

\begin{abstract}
Background. Depressive symptoms are frequent side effects of interferon $\alpha$ therapy (IFN- $\alpha$ ). Both biological and psychological processes may occur concomitantly during hepatitis C treatment.

Objectives. This study was carried out to determine the impact of biological (immune response) and psychological factors on formation of depressive symptoms and major depressive disorder (MDD) during hepatitis C treatment.
\end{abstract}

Material and methods. A total of 99 patients receiving pegylated IFN- $\alpha$ and ribavirin for chronic C type hepatitis participated in the prospective cohort study. Symptoms of depression were assessed with the Montgomery-Åsberg Depression Rating Scale (MADRS) during treatment and 24 weeks after treatment. Neuroticism was measured with the Eysenck Personality Questionnaire - Revised (EPQ-R/N). Diagnosis of MDD was made using the Present State Examination (PSE-10) and DSM-IV-TR criteria. Factor analysis was used to detect factors adding up to total severity of depressive symptoms. Predictors of MDD were investigated using logistic regression analysis.

Results. Factor analysis returned 3 factors: $1^{\text {st }}-$ MADRS scores at weeks $0-12,2^{\text {nd }}-$ MADRS and N scores before treatment, $3^{\text {rd }}-$ MADRS at the $24^{\text {th }}$ week of treatment and 24 weeks after treatment. The total severity of depressive symptoms consisted of 3 components: personality-related before treatment, IFN- $\alpha$-related during treatment and dependent on the effect of treatment. Regression analysis showed that a history of psychiatric disorders $(O R=4.8)$ and MADRS scores before treatment $(O R=1.25)$ were predictors of $M D D$, as opposed to level of neuroticism.

Conclusions. The severity of depressive symptoms and MDD during the hepatitis C treatment was related to general depressive vulnerability, not to psychological factors related to neuroticism.

Key words: depression, hepatitis C, interferon $\alpha$, neuroticism trait 


\section{Introduction}

Interferon $\alpha$ (IFN- $\alpha)$ in combination with ribavirin used to be the main pharmacological treatment for the chronic hepatitis $\mathrm{C}$ virus ( $\mathrm{HCV}$ ) infection. Interferon $\alpha$ is known to induce several neuropsychiatric side effects including depression, anxiety, psychosis, hypomanic mood, and cognitive impairment. ${ }^{1}$ Depression is a particularly common side effect and in some rare cases it may be associated with suicidal ideation or suicide attempts. ${ }^{2}$ Previous psychiatric history was a strong risk factor for depression, anxiety and other psychiatric disorders during treatment, which might suggest that the overall susceptibility to depression and anxiety, including susceptibility of a psychological origin, underlay neuropsychiatric side effects induced by IFN- $\alpha$.

Interferon- $\alpha$-induced neuropsychiatric symptoms have been attributed to the release of pro-inflammatory cytokines modulating several neurophysiological and neuroendocrine systems involved in mood regulation. ${ }^{3,4}$ This is a multidirectional action involving neurotransmitter systems in the central nervous system (CNS), biochemical changes in the CNS and in the hypothalamic-pituitary-adrenal (HPA) axis. Interferon $\alpha$ is a potent inducer of pro-inflammatory cytokines including interleukins 1 and 6 (IL-1, IL-6) and tumor necrosis factor $\alpha$ (TNF- $\alpha$ ). These cytokines play an important role in the development of sickness behavior - a set of psychological and behavioral changes that may lead to depressive symptoms in approx. half of the patients treated with IFN- $\alpha$. The presumably depressogenic effect is not directly caused by cytokines but is a consequence of sickness behavior. This cytokineinduced behavioral syndrome is associated with alterations in the metabolism of neurotransmitters such as serotonin, norepinephrine and dopamine in brain regions essential to the regulation of emotions, including the limbic system, as well as the regulation of psychomotor and reward functions, including the basal ganglia. ${ }^{3-6}$ Cytokines activity is associated with: 1) significant alterations in diurnal HPA axis activity including the flattening of the adrenocorticotropin (ACTH) and cortisol diurnal fluctuations, and an increase in evening $\mathrm{ACTH}$ and cortisol concentrations ${ }^{4}$; 2) increased activity of the metabolic enzyme indoleamine 2,3-dioxygenase responsible for degrading tryptophan to kynurenine which is then metabolized to quinolinic acid interfering with $\mathrm{CNS}$ activity ${ }^{7}$; 3) decreased brainderived neurotrophic factor (BDNF) levels ${ }^{8}$, and 4) altered function of the glucocorticoid receptors. ${ }^{9}$

The effect of IFN- $\alpha$ seems to be purely biological rather than psychological, but patients during an antiviral treatment are going through a difficult phase in their life caused by neuropsychiatric symptoms such as emotional lability, cognitive decline and insomnia, as well as somatic symptoms including fever, nausea, lack of appetite, and weakness. In vulnerable individuals, this stressful situation may cause depression. Significantly, both biological and psychological processes may occur concomitantly during hepatitis $\mathrm{C}$ treatment; in this light, the present paper offers an analysis of their mutual interdependence.

\section{Material and methods}

\section{Participants and treatment}

A total of 99 patients (50 men and 49 women) were included in the study. All of them had a chronic HCV infection with a detectable serum HCV-RNA concentration and compensated liver disease. The patients were scheduled for treatment with pegylated IFN- $\alpha$ and ribavirin in the Ward of Infectious Diseases at the Department of Infectious Diseases, Liver Diseases and Acquired Immune Deficiences at Wroclaw Medical University, Poland. All participants were over 18 years of age and were recruited from native Polish populations in the Lower Silesian region. The inclusion scheme was based on the order in which the patients had showed up for hepatitis $C$ treatment, with sex stratification used to compensate for the number of men and women in the study group. The exclusion criteria entailed comorbidity with severe somatic disease, autoimmune diseases, neurological disorders (including dementia or brain injury), substance dependence (except for nicotine), active psychotic disorders, depression, and pregnancy. The study protocol was approved by the Wroclaw Medical University Ethics Committee. All patients provided their written informed consent.

Initially, the subjects were receiving weekly doses of $180 \mu \mathrm{g}$ Pegintron ${ }^{\circledR}$ (PEG-IFN-a2a, Hoffmann-LaRoche, Basel, Switzerland) combined with either 1,000 mg/day or $1,200 \mathrm{mg} /$ day of Rebetol (ribavirin, Schering-Plough Corporation, Kenilworth, USA), depending on their body weight (those weighing $75 \mathrm{~kg}$ and more were administered the higher dose). Except for patients infected with genotype 3 virus, who were treated for 24 weeks; in the case of all other subjects the treatment lasted 48 weeks.

\section{Psychiatric assessments}

The study followed a prospective longitudinal cohort design. The subjects were evaluated before the treatment (week 0), at weeks $2,4,8,12$, and 24 , and 24 weeks after the conclusion of the treatment. Because patients with $\mathrm{HCV}$ genotype 3 were treated for 24 weeks and the remainder for 48 weeks, assessment point at the end of treatment was omitted in analysis. At each assessment point blood samples were collected and 2 psychometric scales were administered: Montgomery-Åsberg Depression Rating Scale (MADRS) and the Present State Examination (PSE-10). Neuroticism score was assessed once, at the beginning of the study, with the Eysenck Personality Questionnaire - Revised (EPQ-R/N). 
The MADRS scale consists of 10 items assessing symptoms associated with depression, each item scored from 0 to 6 according to severity. ${ }^{10}$ We used the MADRS scale as adapted by Mazurek et al., which proved its good psychometric properties. ${ }^{11}$ The PSE-10 scale is a part of the Schedules for Clinical Assessment in Neuropsychiatry (SCAN 2.1) - a set of instruments and manuals aimed at assessing, measuring and classifying psychopathology and behavior associated with major psychiatric disorders of adult life. ${ }^{12}$ We utilized only sections 6 and 7 of the PSE-10 (i.e., depressed mood and ideation, dysthymia, recurrent brief depressive disorder and thinking, concentration energy, interests), adopting time criteria for depression. The Polish adaptation of SCAN was a collateral result of the international EDEN (European Day Hospital Evaluation) project and as such was successfully tested in several clinical applications. ${ }^{13-15}$ The PSE-10 served as a diagnostic tool for depression according to the DSM-IV criteria at all time points. Psychiatric disorders prior to treatment were assessed by a psychiatrist following a clinical interview. The scale of neuroticism was derived from the EPQ-R questionnaire. It consists of 24 items referring to a minor emotional distress. Neuroticism is an indicator of vulnerability to depression understood as a reaction to stressful life events. ${ }^{16-18}$ We used the Polish adaptation of the EPQ-R authored by Brzozowski and Drwal. ${ }^{19}$

Depressive symptoms resulting from stressful life events are the effect of the interaction between the burden of stressful life events (SLE) and neuroticism level (N). ${ }^{17}$ Depressive symptoms due to biological processes are not dependent on personality structure, and thus are not related to neuroticism level. We assumed that the severity of depressive symptoms is the sum of 2 components: the biological and psychological. Therefore, part of the variation of depressive symptoms related to neuroticism can be considered to be of psychogenic origin, while the remainder of the variance of depressive symptoms - independent of neuroticism - of a biological one.

The subjects had a diagnosed baseline mental condition, and their psychiatric history was evaluated by a psychiatrist. None of the participants were receiving any antidepressant or anxiety medications within 6 months prior to inclusion in the study. Antidepressant prescription during the study, if introduced, was dictated by the clinical judgment of the responsible physician and was not further controlled with study protocol. Nevertheless, concomitant medications including antidepressants were recorded by study personnel at each assessment point.

\section{Statistics}

To analyze the relationships between the severity of depressive symptoms at different time points, the principal components factor analysis method with varimax rotation was used. This method detects the heterogeneity of the depressive process and enables its further investigation in the biological (IFN) and psychological (neuroticism, response to treatment effects) context.

The logistic regression/stepwise model method was used to determine predictors for major depressive disorder (MDD). Demographic, biological and psychiatric variables were included in the marginal results table using logistic regression forward stepwise procedure with sigma-restricted parameterization for categorical predictors. The dependent MDD variable was coded as MDD diagnosis for a good code and no MDD for a bad code. Variables which reached a statistically significant impact on the accuracy of a discriminative function were included in the analysis. All the statistical computations were performed with Dell STATISTICA v. 13.1 for Windows (Microsoft, Armonk, USA).

\section{Results}

Out of 99 patients enrolled, 85 (85.9\%) completed the treatment. The authors decided to perform analysis for MDD predictors in all patients except for 1 who had dropped out after 2 weeks of the treatment. The remainder stayed in treatment for at least 8 weeks which was considered sufficient for depressive symptoms to emerge. Table 1 shows pre-treatment demographic and clinical characteristics of the sample. None of the patients was diagnosed with MDD before the treatment but 14 patients (14.1\%) reached MADRS score of 13 and over, and 4 patients reached MADRS score of 18 and above, which meant that some of them were actually depressed although they did not meet the formal DSM-IV-TR criteria for a major depressive episode. ${ }^{20}$ The initial MADRS mean score in men (5.7) was slightly lower than in women (7.0), but the difference was not significant $(t=-1.38, \mathrm{p}=0.17)$. Of the $98 \mathrm{pa}-$ tients $42(42.9 \%)$ met criteria for MDD at any time within the 24 weeks of treatment, women more often (49.0\%) than men (36.8\%), but this difference was not statistically significant $\left(\mathrm{X}^{2}=1.5, \mathrm{p}=0.22\right)$; neither was the difference in mean maximal MADRS score between men (16.4) and women (18.5) significant $(\mathrm{t}=-1.44, \mathrm{p}=0.15)$.

The mean severity of depressive symptoms fluctuated in the course of treatment: it doubled during the first 4 weeks, then remained stable until week 24 , and dropped slightly below the initial level 24 weeks after the treatment (Fig. 1).

In order to assess the structure of depressive symptoms and their association with the level of neuroticism (i.e., psychological factors), factor analysis was performed in 85 (85.9\%) patients that completed the treatment. Eigenvalues of the first 3 factors were $5.62,0.88$ and 0.62 , respectively. The remaining eigenvalues were below 0.35 , so analysis for a model with 3 factors was carried out. Factor analysis (Table 2) separates MADRS scores during treatment (weeks 2, 4, 8, 12, and 24-factor 1) from those after treatment (week 24 of the treatment and the $24^{\text {th }}$ week after 
Table 1. Comparison of pre-treatment demographic and clinical characteristics between patients with and without major depressive disorder during antiviral treatment

\begin{tabular}{|c|c|c|c|}
\hline Baseline & $\begin{array}{c}\text { All } \\
(n=98)\end{array}$ & $\begin{array}{c}\text { No } \\
\text { depression } \\
(n=56)\end{array}$ & $\begin{array}{l}\text { Major } \\
\text { depressive } \\
\text { episode } \\
(n=42)\end{array}$ \\
\hline Male, $n(\%)$ & $49(50)$ & $31(63.2)$ & $18(36.8)$ \\
\hline $\begin{array}{l}\text { Age [years], mean (SD) } \\
\text { range }\end{array}$ & $\begin{array}{c}46.5(11.1) \\
22-68\end{array}$ & $\begin{array}{c}45.6(11.0) \\
24-68\end{array}$ & $\begin{array}{c}47.7(11.2) \\
22-68\end{array}$ \\
\hline $\begin{array}{l}\text { Genotype, n (\%) } \\
1 \\
2 \\
3 \\
4\end{array}$ & $\begin{array}{c}67(68.4) \\
0(0) \\
24(24.5) \\
7(7.1)\end{array}$ & $\begin{array}{c}39(69.7) \\
0(0) \\
12(21.4) \\
5(8.9)\end{array}$ & $\begin{array}{c}28(66.7) \\
0(0) \\
12(28.6) \\
2(4.7)\end{array}$ \\
\hline $\begin{array}{l}\text { MADRS, mean (SD) } \\
\text { range }\end{array}$ & $\begin{array}{c}6.5(4.9) \\
0-20\end{array}$ & $\begin{array}{c}4.1(3.1) \\
0-13\end{array}$ & $\begin{array}{l}9.4(4.9) \\
2-20\end{array}$ \\
\hline $\begin{array}{l}\text { Neuroticism, mean (SD) } \\
\text { range }\end{array}$ & $\begin{array}{c}10.0(5.7) \\
0-24\end{array}$ & $\begin{array}{c}7.9(4.8) \\
0-21\end{array}$ & $\begin{array}{c}12.7(5.7) \\
3-24\end{array}$ \\
\hline $\begin{array}{l}\text { Body weight, mean (SD) } \\
\text { range }\end{array}$ & $\begin{array}{c}78.9(16.1) \\
47-126\end{array}$ & $\begin{array}{c}80.1(16.2) \\
47-126\end{array}$ & $\begin{array}{c}77.3(17.3) \\
53-117\end{array}$ \\
\hline Alcohol abuse, n (\%) & $10(10.2)$ & $8(15.8)$ & $2(4.8)$ \\
\hline Substance abuse, n (\%) & $13(13.1)$ & $8(14.2)$ & $5(11.9)$ \\
\hline Smoking, n (\%) & $56(57.1)$ & $34(60.7)$ & $22(52.4)$ \\
\hline Psychiatric history, n (\%) & $22(22.5)$ & $5(8.9)$ & $17(40.5)$ \\
\hline
\end{tabular}

SD - standard deviation.

Table 2. Results of factor analysis with the application of the 3-factor model

\begin{tabular}{|l|c|c|c|}
\hline \multirow{2}{*}{\multicolumn{1}{|c|}{ Variables }} & \multicolumn{3}{|c|}{ Factor loadings } \\
\cline { 2 - 4 } & factor 1 & factor 2 & factor 3 \\
\hline MADRS 0 & 0.55 & 0.72 & 0.05 \\
\hline MADRS 2 & 0.85 & 0.33 & 0.23 \\
\hline MADRS 4 & 0.88 & 0.34 & 0.21 \\
\hline MADRS 8 & 0.86 & 0.14 & 0.40 \\
\hline MADRS 12 & 0.77 & 0.22 & 0.48 \\
\hline MADRS 24 & 0.64 & 0.28 & 0.60 \\
\hline MADRS 24a & 0.28 & 0.16 & 0.90 \\
\hline Neuroticism 0 & 0.16 & 0.91 & 0.24 \\
\hline Explained variance & 3.66 & 1.75 & 1.72 \\
\hline \% of total & 0.46 & 0.22 & 0.21 \\
\hline
\end{tabular}

the treatment - factor 3) and before treatment (neuroticism and MADRS before treatment - factor 2). The association between the severity of depressive symptoms and neuroticism scores observed in this model points towards a psychogenic origin of these symptoms. This association is only significant before the treatment, so the rise of the severity of depressive symptoms during treatment is not related to initial neuroticism level, which is attributable to personality factors. The $1^{\text {st }}$ and most significant factor is a direct consequence of the pro-inflammatory action of IFN- $\alpha$. The $3^{\text {rd }}$ factor included MADRS scores at the $24^{\text {th }}$ week of treatment and $24^{\text {th }}$ week after the treatment. It shows the difference in the depressive response

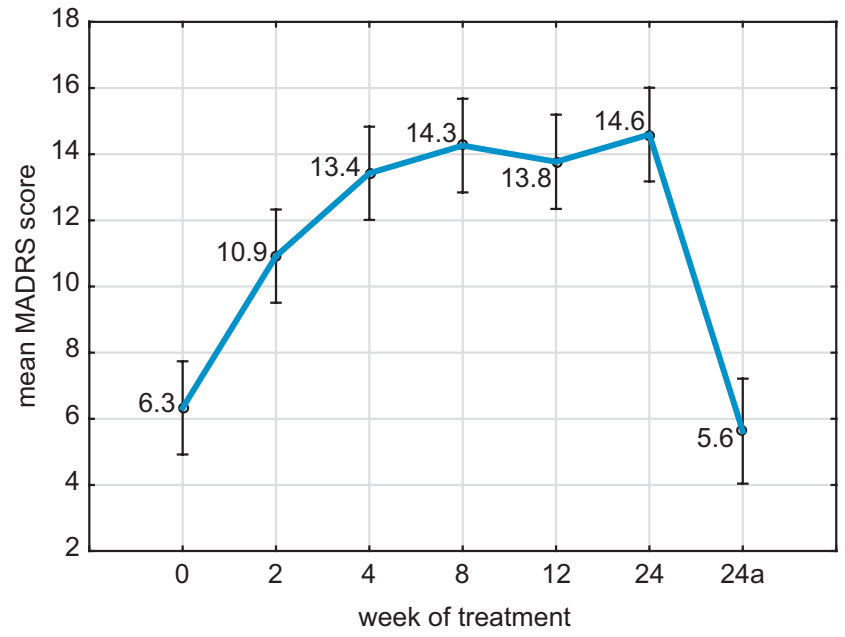

Fig. 1. The average MADRS scores during treatment of hepatitis $C$ with IFN- $\alpha$ and ribavirin

when the treatment came to an end. The severity of depressive symptoms decreased to varying degrees in patients and hence the last 2 measurements were less correlated with other.

To identify MDD predictors, the procedure performed was stepwise logistic regression method with bidirectional elimination testing at each step for variables to be included or excluded. The model included the following variables: age, sex, weight, years of formal education, MADRS score before the treatment, the Eysenck's neuroticism scale score before the treatment, HCV genotype 1,4/2,3, grading, staging before treatment, tobacco smoking, alcohol abuse, psychoactive drugs abuse, family history of psychiatric disorders, and individual history of psychiatric disorders.

Stepwise logistic regression method returned 3 variables that were found to significantly affect the diagnosis of MDD, i.e., the initial MADRS score, the initial neuroticism score and individual history of psychiatric disorders. After these variables had been entered into the logistic regression model, only 2 proved to be statistically significant, i.e., the initial MADRS score and individual history of psychiatric disorders. The results of logistic regression calculations are presented in Table 3.

\section{Discussion}

Shifts in the severity of depressive symptoms during antiviral treatment may appear as a continuous process. Mild symptoms which occur prior to treatment increase during treatment and decrease after treatment. In our cohort study, we managed to capture the diversity of this process. Factor analysis revealed 3 different components of depression changing with time. The main component (factor 1 in the 3 -factor model) relates to IFN action. Depressive symptoms due to IFN administration persisted all through the treatment. The biological impact of IFN 
Table 3. Results of logistic regression analysis

\begin{tabular}{|l|c|c|c|c|}
\hline \multicolumn{1}{c|}{ Effect } & Wald statistics & OR & OR & OR \\
lower CL (95\%) & upper CL (95\%) \\
\hline Intercept & 8.5 & - & - & - \\
\hline MADRS 0 & 8.16 & 1.25 & 1.07 & 0.0035 \\
\hline Neuroticism & 0.46 & 1.04 & 0.93 & 1.46 \\
\hline History of psychiatric disorders & 6.18 & 4.79 & 1.40 & 1.17 \\
\hline
\end{tabular}

OR - odds ratio; CL - confidence limit.

on mood did not depend on psychological factors related to neuroticism. On the other hand, the 2 remaining components, which appear to be much weaker, seemed to be related to psychological factors. The $3^{\text {rd }}$ factor includes depressive symptoms at 2 timepoints, i.e., at the $24^{\text {th }}$ week of the treatment and 24 weeks after the treatment. Therefore, it might be assumed that this component of depression is an effect of stress which in fact corresponds to worries over the outcome of the antiviral treatment - those patients who had recovered were less depressive. Towards the end of treatment, the patients might have already begun to suspect what the results of their treatment would be, and at the endpoint of this study (24 weeks after the treatment) some of them gained certainty - for example, after receiving information about the presence of HCV RNA in blood plasma at the end of treatment. Given that the second factor includes MADRS and neuroticism ratings before treatment, it reflects the psychological reaction to a stressful life situation that the liver disease certainly is. Patients suffering from different somatic diseases are known to have depressive symptoms correlating with the level of their neuroticism: the more severe illness, the stronger the correlation between neuroticism and depression. ${ }^{21}$ Somatic illness as a stressful life situation may cause depressive symptoms in vulnerable individuals, and the neuroticism score is a measure of this vulnerability. ${ }^{22}$ The results of this study did not confirm this relationship - the severity of depressive symptoms before treatment turned out to be a stronger predictor of MDD than the level of neuroticism. This relationship is quite common in other studies on depression in which the initial severity of depressive symptoms was a strong predictor of depressive symptomatology. ${ }^{23-26}$ Depressive symptoms in general seem to be the main predictor of depressive disorders and broad psychiatric and somatic symptomatology. A similar situation has been replicated in the present study - depressive symptoms, despite their origin, foreshadowed MDD in patients treated for hepatitis C. Moreover, the diagnosis of depression during treatment depended solely on the initial severity of depressive symptoms.

In the 3-factor model, the pre-treatment depressive symptoms are almost evenly dispersed between factors 1 (biological) and 2 (psychological). The initial severity of symptoms seems to be a superposition of 2 depressive processes, i.e., the biological one which is related to $\mathrm{HCV}$ infection and evolves considerably during treatment, and the psychological one which is bound to neuroticism ratings and gradually loses its significance. Factor 1 related to the IFN-induced depressive symptoms (46\% of variance) proved much stronger than factor 2 related to neuroticism (22\% of variance); the point of reference for such a distinction is the overall severity of symptoms among which IFN-induced depression predominates. Seemingly, there exists a direct link between pre-treatment depression and IFN-induced depression. The inflammation processes, endogenic cytokines activity and direct effects of administered INF- $\alpha$ constitute the base for this interconnection.

Hepatitis $C$ infection causes a chronic mild inflammation affecting both the liver and the brain, especially its white matter. ${ }^{27,28}$ Hepatitis type $\mathrm{C}$ virus replicates in the mononuclear cells of the immune system and within the brain cells. $^{29,30}$ The inflammation is usually very mild and does not cause noticeable signs of encephalitis, but may still trigger a mild cognitive dysfunction in the form of attention and memory decline observable in the results of neuropsychological cognitive tests. Moreover, the inflammation may also be detected with the use of sophisticated neuroimaging methods such as the computed tomography diffusion tensor. ${ }^{27,31}$ It is very likely that IFN-induced cytokines activity during the treatment is the continuation of inflammation-induced cytokines activity from before the treatment. Both may lead to MDD through illness behavior.

The results of the study showed that patients who previously suffered from mental disorders, as well as patients with a high level of depressive symptoms before treatment, are more susceptible to the development of a depressive disorder during treatment with IFN- $\alpha$. Giving more attention to this group of patients will facilitate a rapid implementation of antidepressant treatment, which may prevent cases of treatment discontinuation.

The results of the study suggest that active assessment of the severity of depressive symptoms during control examinations (not only responding to patients' complaints) is necessary to capture the depressive disorder at the subclinical stage, in order to start a more effective treatment.

\section{ORCID iDs}

Krzysztof Małyszczak (D) https://orcid.org/0000-0001-6295-2742 Małgorzata Inglot (D) https://orcid.org/0000-0002-6173-7909 Dorota Frydecka (D) https://orcid.org/0000-0001-8582-9958 Tomasz Hadryś (D) https://orcid.org/0000-0003-4120-7748 Tomasz Pawłowski (D) https://orcid.org/0000-0002-3997-609X 


\section{References}

1. Myint AM, Schwarz MJ, Steinbusch HW, Leonard BE. Neuropsychiatric disorders related to interferon and interleukins treatment. Metab Brain Dis. 2009;24(1):55-68. doi:10.1007/s11011-008-9114-5

2. Capuron L, Miller AH. Cytokines and psychopathology. Lessons from interferon-alpha. Biol Psychiatry. 2004;56(11):819-824. doi: 10.1016/j. biopsych.2004.02.009

3. Raison $\mathrm{CL}$, Capuron L, Miller AH. Cytokines sing the blues. Inflammation and the pathogenesis of depression. Trends Immunol. 2006;27(1): 24-31. doi:10.1016/j.it.2005.11.006

4. Raison CL, Borisov AS, Woolwine BJ, Massung B, Vogt G, Miller AH. Interferon-alpha effects on diurnal hypothalamic-pituitary-adrenal axis activity. Relationship with proinflammatory cytokines and behavior. Mol Psychiatry. 2010;15(5):535-547. doi:10.1038/mp.2008.58

5. Dunn AJ, Wang J, Ando T. Effects of cytokines on cerebral neurotransmission. Comparison with the effects of stress. Adv Exp Med Biol. 1999;461:117-127. doi:10.1007/978-0-585-37970-8 8

6. Felger JC, Miller AH. Cytokine effects on the basal ganglia and dopamine function: The subcortical source of inflammatory malaise. Front Neuroendocrinol. 2012;33(3):315-327. doi:10.1016/j.yfrne.2012.09.003

7. Baranyi A, Meinitzer A, Breitenecker RJ, Ghadikolai OA, Stauber R, Rothenhäusler HB. Quinolinic acid responses during interferon-ainduced depressive symptomatology in patients with chronic hepatitis $C$ infection: A novel aspect for depression and inflammatory hypothesis. PLoS One. 2015;10(9):e0137022. doi:10.1371/journal.pone. 0137022

8. Lotrich FE, Albusaysi S, Ferrell RE. Brain-derived neurotrophic factor serum levels and genotype: Association with depression during interferon-a treatment. Neuropsychopharmacology. 2013;38(6): 985-995. doi:10.1038/npp.2012.263

9. Udina M, Navinés R, Egmond E, et al. Glucocorticoid receptors, brainderived neurotrophic factor, serotonin and dopamine neurotransmission are associated with interferon-induced depression. Int J Neuropsychopharmacol. 2016;19(4):1-12. doi:10.1093/ijnp/pyv135

10. Montgomery SA, Asberg M. A new depression scale designed to be sensitive to change. Br J Psychiatry. 1979;134:382-389.

11. Mazurek J, Kiejna A, Małyszczak K, Stępień A. Assessment of depression scales reliability in the Polish population: The Bech-Rafaelsen Melancholia Scale, the Hamilton Depression Scale, and the Montgomery-Asberg Scale. Post Psychiatr Neurol. 1999;8(2):165-172.

12. Wing JK, Babor TT, Brugha TT, et al. SCAN: Schedules for Clinical Assessment in Neuropsychiatry. Arch Gen Psychiatry. 1990;47(6): 589-593. doi:10.1001/archpsyc.1990.01810180089012

13. Kiejna A, Kallert TW, Rymaszewska J. Treatment in psychiatric day hospital in comparison with inpatient wards in different European health care systems - objectives of EDEN project. Psychiatr Pol. 2002; 36(6 Suppl):361-367.

14. Małyszczak K, Rymaszewska J, Hadryś T, Adamowski T, Kiejna A. Comparison between a SCAN diagnosis and a clinical diagnosis. Psychiatr Pol. 2002;36(Suppl 6):377-380.

15. Adamowski T, Kiejna A, Hadryś T. Study of compatibility of psychiatric diagnoses with ICD-10 diagnostic criteria using the SCAN questionnaire. Psychiatr Pol. 2006;40(4):761-773.

16. Eysenck HJ, Eysenck SBG. Manual of the Eysenck Personality Inventory. London, UK: Hodder \& Stoughton; 1975.
17. Kendler KS, Kuhn J, Prescott CA. The interrelationship of neuroticism, sex, and stressful life events in the prediction of episodes of major depression. Am J Psychiatry. 2004;161:631-636. doi:10.1176/appi.ajp. 161.4.631

18. Ormel J, Oldenhinkel AJ, Vollebergh W. Vulnerability before, during, and after a major depressive episode: A 3-wave population-based study. Arch Gen Psychiatry. 2004;61(10):990-996. doi:10.1001/archpsyc.61.10.990

19. Brzozowski P, Drwal RŁ. Eysenck Personality Questionnaire, Polish adaptation of EPQ-R. Pracownia Testów Psychologicznych PTP, Warszawa, Poland; 1995.

20. Sagen U, Vik TG, Moum T, Mørland T, Finset A, Dammen T. Screening for anxiety and depression after stroke: Comparison of the hospital anxiety and depression scale and the Montgomery and Asberg depression rating scale. J Psychosom Res. 2009;67(4):325-332. doi:10. 1016/j.jpsychores.2009.03.007

21. Jylhä P, Melartin T, Isometsä E. Relationships of neuroticism and extraversion with axis I and II comorbidity among patients with DSM-IV major depressive disorder. J Affect Disord. 2009;114(1-3):110-121. doi:10.1016/j.jad.2008.06.011

22. Ormel J, Jeronimus BF, Kotov R, et al. Neuroticism and common mental disorders: Meaning and utility of a complex relationship. Clin Psychol Rev. 2013;33(5):686-697. doi:10.1016/j.cpr.2013.04.003

23. Choi JS, Kim W, Sohn BK, et al. Association of changes in mood status and psychosocial well-being with depression during interferon-based treatment for hepatitis C. Psychiatry Investig. 2017;14(3): 314-324. doi:10.4306/pi.2017.14.3.314

24. Mahajan S, Avasthi A, Grover S, Chawla YK. Role of baseline depressive symptoms in the development of depressive episode in patients receiving antiviral therapy for hepatitis C infection. J Psychosom Res. 2014;77(2):109-115. doi:10.1016/j.jpsychores.2014.05.008

25. Carta MG, Hardoy MC, Garofalo A, et al. Association of chronic hepatitis $C$ with major depressive disorders: Irrespective of interferon-alpha therapy. Clin Pract Epidemiol Ment Health. 2007;3:22. doi:10.1186/17450179-3-22

26. Franco FGM, Laurinavicius AG, Lotufo PA, et al. Persistent depressive symptoms are independent predictors of low-grade inflammation onset among healthy individuals. Arq Bras Cardiol. 2017;29:0. doi:10. 5935/abc. 20170080

27. Bladowska J, Zimny A, Knysz B, et al. Evaluation of early cerebral metabolic, perfusion and microstructural changes in HCV-positive patients: A pilot study. J Hepatol. 2013;59(4):651-657. doi:10.1016/ j.jhep.2013.05.008

28. Mathew S, Faheem M, Ibrahim SM, et al. Hepatitis $C$ virus and neurological damage. World J Hepatol. 2016;8(12):545-556. doi:10.4254/ wjh.v8.i12.545

29. Forton DM, Karayiannis P, Mahmud N, Taylor-Robinson SD, Thomas HC. Identification of unique hepatitis $C$ virus quasispecies in the central nervous system and comparative analysis of internal translational efficiency of brain, liver, and serum variants. J Virol. 2004;78(10): 5170-5183.

30. Laskus T, Radkowski M, Adair DM, Wilkinson J, Scheck AC, Rakela J. Emerging evidence of hepatitis C virus neuroinvasion. AIDS. 2005; 19(Suppl 3):140-144.

31. Monaco S, Mariotto S, Ferrari S, et al. Hepatitis C virus-associated neurocognitive and neuropsychiatric disorders: Advances in 2015. World J Gastroenterol. 2015;21(42):11974-11983. doi:10.3748/wjg.v21. i42.1197 